\title{
PIERRE BONTE
}

\section{La Méditerranée des anthropologues Permanences historiques et diversité culturelle}

Contrairement à l'Afrique ou encore à l'Amérique et à l'Océanie, la Méditerranée a tardé à s'imposer aux anthropologues comme une aire culturelle justifiant des approches et des institutions particulières: pendant longtemps il n'y a pas eu un champ particulier des »études méditerranéennes«, comme c’est le cas pour les études africanistes, ou américanistes, ou encore océanistes. Il est vrai que la discipline anthropologique s'est constituée à partir d'observations et de travaux effectués initialement sur ces sociétés étrangères à l'Occident où s'imposaient d'autant plus les paradigmes anthropologiques qu'elles étaient plus ou moins exclues de l'histoire universelle hégélienne. $\mathrm{Si}$ les rives européennes relevaient bien de celle-ci, le statut des rives musulmanes, bien qu'elles soient des lieux de hautes civilisations, perpétuait de ce point de vue une ambiguité ${ }^{1}$ qui se poursuivra avec la colonisation, creusant les écarts entre pays colonisateurs et pays colonisés. La notion anthropologique d'un ensemble méditerranéen n'émergera de fait qu'après la Seconde Guerre mondiale, et accompagne le mouvement rapide de décolonisation qui suit celle-ci. Les anthropologues étendent parallèlement leur champ d'études, remettant en question le »regard éloigné« qui présidait jusqu'alors à leurs observations: les recherches des anthropologues s'intéressent à la même époque à leurs propres sociétés, au monde rural d'abord, puis urbain, industriel, etc.

\section{HISTOIRE ET ANTHROPOLOGIE}

Ce constat de retard est néanmoins relativement paradoxal si on le rapproche de celui que l'on peut dresser des travaux des géographes ${ }^{2}$ et des historiens qui ont anciennement identifié au sein de cet objet d'étude des permanences qui relèvent alors plus, chez ces derniers, des influences civilisatrices, gréco-romaines, ou judéo-chrétiennes, ou encore celles de l'islam, que de faits relevant de convergences culturelles. Cette opposition entre permanence cumulative des civilisations et diversification des sociétés et cultures va contribuer à brouiller les observations des anthropologues en les focalisant à travers des distinctions rigides: Occident versus Orient, christianisme versus islam, rives européennes versus rives africaines et asiatiques. Elle a façonné la mentali-

1 De cette ambiguïé témoigne l'appellation d'Afrique »blanche«, longtemps attribuée à toute la partie méridionale de la Méditerranée, par opposition à l'Afrique »noire«.

2 L'intérêt des géographes pour la Méditerranée remonte aux auteurs arabes (al-Idrīsī en particulier au $\mathrm{XII}^{\mathrm{e}}$ siècle). Pour un bilan récent des approches géographiques sur le bassin méditerranéen, on consultera Jacques BETHEMONT, Géographie de la Méditerranée, Paris 2001. 
té des voyageurs du XIX ${ }^{\mathrm{e}}$ siècle en même temps que se constituaient de nouveaux paradigmes scientifiques, ceux en particulier de l'orientalisme ${ }^{3}$. Les frontières ainsi tracées n'ont cependant jamais été totalement rigides: le voyage en Italie ou en Grèce est le prélude au voyage en Orient; la pérennité des paysages et des ruines rappelle la relative unité des civilisations passées. Le romantisme produit dans les arts et les lettres du $\mathrm{XIX}^{\mathrm{e}}$ siècle des images d'une Méditerranée réinventée qui s'imposent à l'imaginaire occidental. Ces oppositions produisent néanmoins encore leurs effets dont témoignent les débats contemporains sur l'appartenance de la Turquie à l'Europe.

Si la perspective historique a parfois brouillé le travail comparatif de l'anthropologue, il faut reconnaître qu'elle a aussi finalement contribué - en France du moins à débloquer celui-ci et qu'elle a participé du regard plus totalisant que, depuis une cinquantaine d'années, la discipline porte sur cet objet »Méditerranée«. Deux écoles historiques ont plus particulièrement contribué à cette évolution:

- la première est illustrée par l'ouvrage majeur de Fernand Braudel, »La Méditerranée et le monde méditerranéen à l'époque de Philippe II«, paru en 1949, qui porte un regard nouveau sur la Méditerranée à l'aube des Temps modernes et qui illustrera la constitution de l'école des »Annales«. La démarche >relationnelleく, mettant l'accent sur les échanges matériels et immatériels, et globalisante, incite à un approfondissement des recherches comparatives dans le champ du social et du culturel qui ne sera pas sans conséquences sur les recherches anthropologiques;

- la seconde est celle des antiquisants, qui s'inspirent des concepts et méthodes de l'anthropologie pour aborder l'étude des sociétés anciennes du bassin méditerranéen, la Grèce des cités en particulier. Il en résultera, pour s'en tenir à la France, une série de publications, en continuité avec les travaux précurseurs de Louis Gernet ${ }^{4}$, d'auteurs tels que Jean-Pierre Vernant, Pierre Vidal-Naquet, Nicole Loraux, Marcel Detienne, etc., se revendiquant d'une anthropologie historique. Ces auteurs s'engagent dans des comparaisons avec les sociétés contemporaines 5 et ils collaborent parfois directement avec les anthropologues sur des programmes ouverts aux deux disciplines, tel celui sur le polythéisme organisé par le Centre national de la recherche scientifique dans les années 1980.

Ces passerelles épistémologiques et méthodologiques entre histoire et anthropologie ne sont pas sans avoir des conséquences plus générales sur les recherches orientalistes, amenant à une progressive remise en question des oppositions sur lesquelles elles se

3 La constitution de l'orientalisme comme discipline scientifique a largement contribué aux études méditerranéennes, en particulier les premières recherches sur l'Égypte au début du $\mathrm{XIX}^{\mathrm{e}}$ siècle. La démarche orientaliste se définit elle-même à travers une série d'oppositions: écrit versus oral, savant versus populaire, grande versus petite tradition, etc. Elle aborde essentiellement les faits culturels à travers les langues et dessine un champ historicisé des études locales qui ne laissera qu'une place résiduelle à une ethnologie qui s'intéresse aux faits d'oralité, à la culture populaire, etc.

4 Louis GERNET, Droit et société dans la Grèce ancienne, Paris 1955 (Publications de l'institut de droit romain de l'université de Paris, 13).

5 À propos des rituels sacrificiels dans le monde grec ancien et moderne, voir Marcel DETIENNE, Jean-Pierre VERNANT, La cuisine du sacrifice en pays grec, Paris 1979. 
fondaient, telles que celles entre Occident et Orient ${ }^{6}$, Indo-Européens et Sémitiques, etc. Les conséquences, faut-il le souligner, sont parfois imprévues. Louis Gernet, historien à l'œuvre duquel se réfèrent les hellénistes français contemporains, publie dès avant la Première Guerre mondiale dans "L'Année sociologique«. Professeur à l'université d'Alger, il refuse toute affectation en France - fût-elle assortie d'une promotion - et engage une réflexion sur le parallélisme entre les rituels grecs anciens, les frairies paysannes par exemple, et les rituels sacrificiels collectifs maghrébins. Il alimente en ce sens, et sans doute bien involontairement, les thèses d'une ethnologie >coloniale < française qui particularise la berbérité en la rapprochant d'un fond institutionnel ${ }^{7}$ ou rural méditerranéen ${ }^{8}$, et qui oppose la tradition occidentale autochtone de la population maghrébine et les apports orientaux - arabes et musulmans -, sources de régression?.

Le développement du comparatisme et le rapprochement de données historiques et contemporaines ne sont donc pas sans soulever de problèmes. Ils subvertissent cependant progressivement les paradigmes de l'orientalisme en investissant le champ méthodologique de celui-ci centré sur l'archéologie et la philologie. Une relecture des textes fondateurs s'inspire des travaux anthropologiques sur les mythes chez les hellénistes ou encore chez Jean Bottero ${ }^{10}$ dans le cas de la Mésopotamie. L'intérêt pour cette démarche est d'autant plus grand quand il s'agit de la relecture des textes sacrés des trois religions révélées entre lesquelles se répartissent les Méditerranéens contemporains: cette relecture relève des motifs communs ${ }^{11}$ et conduit à de nouvelles analyses qui réduisent l'écart entre religion savante et religion populaire et rapprochent les traditions culturelles au-delà de leurs distinctions figées ${ }^{12}$. Ces recherches sur les textes

6 Jack GoODY, L'Orient en Occident, Paris 1999.

7 Émile MASQUERAY, La formation des cités chez les populations anciennes de l'Algérie. Kabyles du Djurdjura, Chaouias de l'Aurès, Beni Mzâb, Paris 1896.

8 Jean SERVIER, Les portes de l'année. Rites et symboles. L'Algérie dans la tradition méditerranéenne, Paris 1962.

9 Cette régression aurait pour cause première les invasions des nomades bédouins hilaliens qui ouvrirait une période de crise des sociétés maghrébines culminant au XVI ${ }^{\mathrm{e}}$ siècle.

10 Jean BotTero, Samuel Noah Kramer, Lorsque les dieux faisaient l'homme. Mythologie mésopotamienne, Paris 1989.

11 Pierre Bonte, Enric Porqueres Y Gene, Jérôme Wilgaux, L'argument de la filiation aux fondements des sociétés européennes et méditerranéennes, Paris 2011.

12 L'évolution des travaux sur le thème indo-européen est à cet égard significative. Ils se sont enrichis des recherches dumeziliennes sur le schéma cosmique tripartite des fonctions, conçu comme le modèle des représentations du monde dans un ensemble dont les contours sont encore linguistiques. Les travaux récents s'ouvrent à une grande diversité de thèmes anthropologiques (voir Émile BENVENISTE, Le vocabulaire des institutions indo-européennes, Paris 1969; Bruce LinColn, Myth, Cosmos and Society: Indo-European Themes of Creation and Destruction, Cambridge 1986; Bernard SERgent, Les Indo-Européens, Paris 1995 et aboutissent à la remise en question de l'opposition héritée de l'histoire scientifique entre indo-européen et sémitique, voir Jean LAMBERT, Le dieu distribué. Une anthropologie comparée des monothéismes, Paris 1995. 
religieux, prolongées par celles sur l'écriture et les comptes ${ }^{13}$, conjuguant approches civilisationnelle et comparatisme, social et culturel, largement centré sur l'aire méditerranéenne, contribuent aussi à redéfinir les frontières disciplinaires entre histoire, orientalisme et anthropologie, et à faire émerger une Méditerranée des anthropologues.

\section{LA MÉDITERRANÉE DES ANTHROPOLOGUES}

Celle-ci prend forme depuis moins d'une cinquantaine d'années et, même si j'ai privilégié dans ces premières pages les travaux liminaires d'auteurs français, elle est surtout l'œuvre d'anthropologues de tradition britannique (d'origine ou de formation). Moins préoccupés de rapprochements avec l'histoire, les anthropologues britanniques, et pour certains nord-américains, ont commencé, au lendemain de la Seconde Guerre mondiale, à appliquer à des terrains méditerranéens les grands principes de la démarche anthropologique: monographie et observation participante. Les itinéraires personnels ont souvent joué dans ce choix; il faut noter que, dès le départ, des anthropologues autochtones ont été concernés par cette démarche: Julio Caro Baroja en Espagne, Ernesto de Martino en Italie, John G. Peristiany en Grèce (d'origine grecque, il a étudié à Oxford et fondé en 1959 le Centre athénien de sciences sociales), etc.

Les premiers travaux de synthèse, ceux de Julian Pitt-Rivers ${ }^{14}$ et de Peristiany ${ }^{15}$, se placent encore sous l'étiquette de la sociologie et concernent surtout les sociétés rurales, se revendiquant du courant ruraliste qui s'affirme alors, dans une tradition ouverte en France par des historiens ${ }^{16}$ et qui a tôt concerné la Méditerranée ${ }^{17}$. Les terrains des anthropologues se multiplient alors dans les pays méditerranéens et très vite apparaissent les premières tentatives de bilan comparatif qui portent sur des thèmes particuliers. En 1965, Peristiany édite un ouvrage collectif sur le thème qui sera le plus exploré, celui de l'honneur ${ }^{18}$; il contribue aussi à l'étude des structures parentales et familiales ${ }^{19}$. Pitt-Rivers, à partir de son terrain andalou qui conjugue Orient et Occi-

13 Jack GoODY, La raison graphique, Paris 1979; ID., La logique de l'écriture. Aux origines des sociétés humaines, Paris 1987; ou encore Clarisse HERRENSCHMIDT, Les trois écritures. Langue, nombre, code, Paris 2007.

14 Julian PitT-Rivers, Mediterranean Countrymen. Essays in the social anthropology of the Mediterranean, La Haye 1963 (Recherches méditerranéennes/Études, 1).

15 John G. Peristiany (dir.), Contributions to Mediterranean Sociology. Mediterranean Rural Communities and Social Change. Acts of the Mediterranean sociological conference, Athens, July 1963, Paris, La Haye 1968 (Publications of the Social sciences centre Athens, 4).

16 Marc BLOCH, Les caractères originaux de l'histoire rurale française, Oslo 1931 (Instituttet for Sammenlignende Kulturforskning, 19).

17 Charles Parain, La Méditerranée. Les hommes et leurs travaux, Paris 1936 (Géographie humaine, 8).

18 John G. Peristiany, Julian PitT-Rivers (dir.), Honor and Grace in Anthropology, Cambridge 1992 (Cambridge Studies in social and cultural anthropology, 76); John G. PERISTIANY (dir.), Honour and Shame. The Values of the Mediterranean Society, Londres 1965.

19 ID. (dir.), Mediterranean Family Structures, Cambridge 1976 (Cambridge Studies in social anthropology, 13). 
dent, islam et christianisme, histoire et anthropologie, aborde pour sa part l'étude du traitement de la notion de genre ${ }^{20}$.

À ma connaissance l'ouvrage de John Davis, paru en 1977, „Peoples of the Mediterranean. An Essay in Comparative Social Anthropology«, représente la première tentative de balayage totalisant du champ de cette anthropologie de la Méditerranée. Davis inventorie les différents développements thématiques de l'anthropologie des peuples de la Méditerranée et met en avant leur cohérence d'ensemble; cette tentative restera cependant une exception et peu d'auteurs s'y risqueront réellement. Néanmoins, l'anthropologie de la Méditerranée acquiert ses lettres de noblesse chez les auteurs anglo-américains et fait progressivement école en France: moins de dix ans plus tard, Bernard Kayser publie dans ce pays un recueil de textes choisis qui souligne les apports des auteurs britanniques et américains ${ }^{21}$.

La définition d'un objet »Méditerranée«, de fait, ne va pas de soi et soulève des critiques dont, par exemple, Michael Herzfeld se fait l'interprète ${ }^{22}$ et auxquelles font écho les participants à un volumineux ouvrage-bilan plus récent qui témoigne par ailleurs de la réappropriation de l'objet par l'anthropologie française ${ }^{23}$.

Si nous la comparons avec l'organisation du champ de l'africanisme par exemple, la constitution des études méditerranéennes obéit à des logiques assez différentes. La recherche africaniste s'est forgée à partir de grandes questions qui ont contribué à la définition de la discipline anthropologique elle-même: diffusionnisme et évolutionnisme, sociétés `sans〈 histoire, écriture, État, rôle de la parenté et en particulier de la filiation unilinéaire, mythes et rites, magie et sorcellerie, etc., questions auxquelles se référent les principales thématiques qui ont été illustrées de manière paradigmatique sur ces terrains africains - sociétés lignagères et segmentaires, rituels sacrificiels, royautés sacrées, etc.

Les études méditerranéennes se sont constituées de manière beaucoup plus agrégative, dans le champ d'une anthropologie déjà constituée dans ses méthodes »observation participante« et ses écoles »théorie de la filiation« versus »théorie de l'alliance«; »anthropologie culturelle« versus »anthropologie sociale«. Elles ont opéré sur la base de thématiques particulières qui ont fait l'objet de développements distincts, ce qui soulève inévitablement le problème de leur cohérence, celle-ci étant ici entendue du point de vue de la discipline - dessinent-elles une aire culturelle présentant des traits >discrets ? C'est une question que nous aborderons ultérieurement - mais aussi, au sens où l'entend Louis Dumont: du point de vue des sociétés qui sont rassemblées sous l'étiquette >méditerranéennes〈. La réponse à cette dernière interrogation est d'autant

20 Julian PitT-Rivers, The People of the Sierra, Londres 1954; ID., The Fate of Shechem or the Politics of Sex. Essays in the Anthropology of the Mediterranean, Cambridge 1977 (Cambridge Studies in social anthropology, 19).

${ }^{21}$ Bernard KAYSER, Les sociétés rurales de la Méditerranée. Un recueil de textes anthropologiques anglo-américains, Aix-en-Provence 1986.

22 Michael Herzfeld, Anthropology Through the Looking Glass. Critical Ethnography in the Margins of Europe, Cambridge 1987.

23 Albera Dionigi, Anton Blok, Christian Bromberger (dir.), Anthropologie de la Méditerranée, Paris 2001. 
plus difficile que ces sociétés sont inscrites dans des ensembles plus vastes, économiques ${ }^{24}$, politiques (empires ${ }^{25}$ ), religieux (grandes religions révélées ${ }^{26}$ ), etc. L'agrégation des traits culturels, mis en évidence à travers ces thématiques privilégiées dont les anthropologues ont abordé l'étude, ne garantit nullement la cohérence qui résulterait de l'organisation de ces sociétés méditerranéennes particulières sous des valeurs communes, partagées ${ }^{27}$. Il se trouve toujours immanquablement quelque contre-exemple qui puisse dénier leur participation à la définition d'une spécificité méditerranéenne plus générale et souligner leurs particularités ${ }^{28}$.

Malgré ces difficultés, des auteurs ont tenté de définir de manière rigoureuse l'unité de la Méditerranée. L'ouvrage de John Davis, qui transcende cette approche agrégative, est une exception mais, malgré un notable effort de clarification, il oscille entre des généralités de faible valeur heuristique et des allégations susceptibles d'être démenties par les faits ${ }^{29}$. Si les auteurs britanniques se satisfont volontiers d'une approche pragmatique qui reconstruit des objets méditerranéens partiels, les auteurs américains vont faire appel à un cadre théorique plus ambitieux. La notion d'»aire culturelle«, de tradition allemande et inspirée du diffusionnisme de ses fondateurs, Leo Frobenius, Fritz Gräbner et le père Wilhelm Schmidt, est passée dans l'anthropologie culturelle américaine, par l'intermédiaire de Franz Boas, Alfred Kroeber et Clark Wissler. Au lendemain de la Seconde Guerre mondiale, les recherches sur le terrain méditerranéen sont cependant rassemblées dans ce cadre par Conrad M. Arensberg, qui

${ }^{24}$ C'est le cas des vastes systèmes commerciaux continentaux qui, jusqu'au Moyen Âge, lient le bassin méditerranéen au reste de l'Asie, la route de la soie en particulier avec la Chine, ou à l'Afrique subsaharienne le long de la route caravanière de l'or soudanais. Ces systèmes commerciaux ont favorisé la circulation des biens, des idées et des hommes à une grande échelle. Les routes maritimes joueront un rôle identique et s'imposeront à partir des $\mathrm{XV}^{\mathrm{e}}$ et $\mathrm{XVI}^{\mathrm{e}}$ siècles.

${ }^{25}$ Les empires romain, puis byzantin, en partie réoccupé par l'Empire ottoman jusqu'au début du $\mathrm{XX}^{\mathrm{e}}$ siècle, ont favorisé aussi le brassage humain et culturel.

26 Après avoir longtemps privilégié les fractures, l'étude des grands monothéismes méditerranéens - judaïsme, christianisme et islam - insiste désormais sur leurs convergences et divergences à partir d'un fonds commun textuel et en partie rituel.

27 Les sociétés tribales que j'ai plus particulièrement étudiées présentent ainsi nombre de traits communs (parenté, alliance de mariage, protection, honneur, etc.), relevant des grandes thématiques des études méditerranéennes, mais elles participent aussi d'ensembles au sein desquels elles développent des traits distinctifs, selon qu'elles s'inscrivent ou non dans des structures étatiques, qu'elles présentent ou non des hiérarchies statutaires et politiques, qu'elles développent des fonctions religieuses ou politico-militaires, etc. Leur cohérence particulière ne résulte pas seulement des traits communs qu'elles partagent, mais aussi de leur inscription dans ces ensembles économiques (systèmes commerciaux), politiques (État), religieux (islam) plus larges.

28 D'autres découpages peuvent être définis, dessinant d'autres ensembles susceptibles de se voir attribuer de nouvelles significations du fait de leurs implications idéologiques et politiques contemporaines: c'est le cas du monde européen ou du monde arabo-musulman, voire sislamique`, restaurant en apparence l'opposition classique Occident/Orient en réintroduisant une dimension civilisationnelle convoquée à l'appui de prétentions politiques et stratégiques, du Grand Moyen-Orient de Georges W. Bush ou de l'Union pour la Méditerranée de Nicolas Sarkozy.

29 John DAVIS, People of the Mediterranean. An Essay in Comparative Social Anthropology, Londres 1977. 
introduit la notion d'une »aire méditerranéenne« dans un article d'»Anthropological Quarterly«, revue qui consacrera plusieurs numéros spéciaux à la question ${ }^{30}$. Cette recherche ne suscitera guère d'adhésion, même si la notion d'aire culturelle a inspiré certains travaux de synthèse, ouvertement ${ }^{31}$ ou de manière plus indirecte. Elle a en effet subi des critiques diverses mais efficaces, dans le contexte de la décolonisation, de la part des anthropologues autochtones en particulier qui y voient une manifestation de l'hégémonie anglo-saxonne dans la discipline. Il faut reconnaître par ailleurs que ses fondements théoriques, diffusionnistes et évolutionnistes, soulèvent encore de nos jours de sérieuses interrogations.

\section{LES PRINCIPAUX THÈMES ABORDÉS}

Effectuons d'abord un inventaire des thématiques abordées par ces études méditerranéennes dont aucune, rappelons-le encore, ne leur est spécifique - certaines l'étant toutefois plus que d'autres comme la problématique de l'honneur.

Techniques et échanges: les travaux en ce domaine, malgré le travail précurseur de Charles Parain ${ }^{32}$ et les apports de la géographie humaine, sont relativement peu développés dans une perspective comparative qui se situerait à l'échelle méditerranéenne. Toutefois on peut citer, entre autres, les recherches menées sur les techniques agraires ${ }^{33}$ et les paysages ${ }^{34}$, sur les systèmes hydrauliques ${ }^{35}$, sur la transhumance animale ${ }^{36}$ et sur l'articulation rural/urbain. Sur ce dernier point, bien qu'ils portent sur le Mexique et non sur le bassin méditerranéen, les travaux de Robert Redfield ont contribué au développement des problématiques méditerranéennes ${ }^{37}$.

30 Conrad M. Arensberg, The Old World Peoples: the Place of European Cultures in World Geography, dans: Anthropological Quarterly 36 (1963), p. 75-99.

31 Louise E. SweEt, Timothy O'LeARY, Circum-Mediterranean Peasantry. Introductory Bibliography, New Haven 1969.

32 PARAIN, La Méditerranée (voir n. 17).

33 Citons à ce propos une nouvelle association consacrant ses activités aux techniques rurales méditerranéennes ATERAM.

34 Ces paysages sont souvent façonnés par l'exploitation complémentaire de niches altitudinales: céréales sur les plaines et plateaux, arboriculture de rapport olivier/vigne, forêt et pâturages d'altitude où l'élevage tient une place importante.

35 Bien que le climat méditerranéen en tant que tel ne relève pas des conditions d'aridité, celles-ci prévalent très vite sur les rives africaines et asiatiques et l'exploitation de ces zones arides a fait l'objet de longue date d'aménagements hydrauliques complexes: oasis et réseaux d'irrigation,

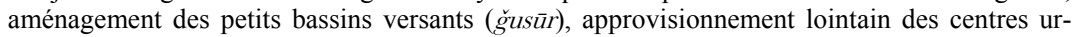
bains, etc. Certaines de ces techniques comme les canaux souterrains des fuggāra-s, dont on trouve les traces anciennes en Iran, ou encore la roue élévatoire, noria, se retrouvent dans une grande partie du bassin méditerranéen.

36 La transhumance animale spécialisée peut conduire à la formation de sociétés pastorales mobiles qui caractérisent une partie de l'aire balkanique méridionale, la Turquie et les steppes et déserts proche-orientaux et nord-africains, ainsi que de nombreuses régions du Maghreb, voir Anne-Marie BRISEBARRE, Bergers et transhumances, Paris 2008.

37 Robert Redfield, The Little Community, Chicago 1955 (The Gottesman Lectures, 5); ID., Peasant Society and Culture, Chicago 1956. 
Parenté et filiation: peu de travaux ont été consacrés à l'analyse des terminologies de parenté dont les convergences n'apparaissent pas de manière évidente ${ }^{38}$. Le statut de la filiation >unilinéaire` privilégié dans la tradition anthropologique britannique, a été interrogé dans les sociétés méditerranéennes. L'accent a été mis principalement sur les sociétés tribales maghrébines et moyen-orientales, privilégiant l'organisation segmentaire et les dynamiques politiques, particulièrement dans le contexte de l'islam ${ }^{39}$. L'existence assez générale de groupes définis par la filiation agnatique (monde arabomusulman) ou utérin (Touaregs, Nubie ancienne, etc.), mais où ces groupes ne présentent pas de traits exogames, qu'il s'agisse des sociétés témoignant du passé méditerranéen (gènos grec, gens romaine...) ou de la situation contemporaine, amènera à déplacer les recherches vers le rôle de la parenté cognatique sindifférenciée` remettant en question les conclusions de la »théorie de la filiation« britannique qui faisait de la filiation unilinéaire un principe général de constitution des groupes sociaux ${ }^{40}$. L'ensemble de la question est repris dans un ouvrage de synthèse ${ }^{41}$. Le rôle de l'adoption a été aussi particulièrement souligné42, avec des effets divers selon les périodes et les cultures juridiques: le droit musulman ne reconnait ainsi que le fosterage et non l'adoption plénière que l'on observe, sous des formes diverses, dans la plupart des cultures anciennes ou chrétiennes. Le fait souligne une autre inflexion que l'on peut constater dans les sociétés méditerranéennes: l'importance de la 'parenté élective< qui obéit à des principes bien différents selon qu'il s'agit de la parenté spirituelle chrétienne ${ }^{43}$ ou de la >parenté de lait`musulmane ${ }^{44}$. Cette flexibilité des représentations et pratiques de la parenté contribue au large usage métaphorique de celle-ci, que l'on observe aussi bien dans le vocabulaire ecclésiastique que dans celui des grou-

38 Des recherches comparatives ont été menées sur les terminologies indo-européennes mais dans une perspective, inspirée de la linguistique historique et fort éloignée du projet anthropologique, de reconstitution d'un système originel proto-européen.

39 Ernest GELLNER, Saints of the Atlas, Londres 1969.

40 Les données méditerranéennes ont contribué à la critique du »modèle lignager et segmentaire« qui faisait de la filiation unilinéaire le modèle des sociétés `sans État< dont l'ordre social ne reposait pas sur l'existence d'institutions politiques spécifiques. Ce modèle africain a été simultanément déconstruit dans les travaux des africanistes, de même qu'a été remis en question son transfert à d'autres sociétés à travers le monde. Pierre BONTE, Édouard CONTE et al., $\mathrm{Al}$ ansab. La quête des origines. Anthropologie historique de la société tribale arabe, Paris, Cambridge 1991; voir aussi Guideon KRESSEL, Descent Trough Males, Wiesbaden 1992 (Mediterranean Language and Culture monograph series, 8) (recueil d'articles plus anciens).

41 Bonte, Porqueres Y Gene, WilgauX, L'Argument de la filiation (voir n. 11).

42 Agnès Fine, Adoption. Ethnologie des parentés choisies, Paris 1998 (Collection droit et société, 29).

43 EAD., Parrains, marraines: la parenté spirituelle en Europe, Paris 1994; Salvatore D’ONAFRIO, L'esprit de la parenté: Europe et horizon chrétien, Paris 2004, et aussi des ouvrages allemands sur la thématique, p.ex. Bernhard JUSSEN, Patenschaft und Adoption im frühen Mittelalter. Künstliche Verwandtschaft als soziale Praxis, Göttingen 1991 (Veröffentlichungen des MaxPlanck-Instituts für Geschichte, 98).

44 Le droit musulman reconnaît un lien, analogue à celui de la consanguinité et créant de même des interdits matrimoniaux, entre deux personnes qui ont bu le lait d'une même nourrice. Cette règle, très respectée dans la pratique, s'explique par une certaine coïncidence des effets des apports de sperme et de lait dans la conception et la fabrication de l'enfant. 
pements mafieux, ou encore dans les multiples formes, plus ou moins extensives, de >fraternités‘, professionnelle, politique, religieuse.

Alliance de mariage: du point de vue de l'alliance de mariage, c'est-à-dire des règles sociales qui président au choix des conjoints, les sociétés méditerranéennes, historiques ou contemporaines, s'inscrivent difficilement dans le cadre des théories anthropologiques - c'est le cas tout particulièrement du »mariage arabe«, entre enfants de frères ${ }^{45}$ - tout en présentant une certaine diversité apparente de forme. Toutefois, nombre d'entre elles expriment une préférence matrimoniale pour le mariage »dans un degré rapproché« de consanguinité ${ }^{46}$. Ces mariages proches qui peuvent impliquer une forte proximité consanguine entre les conjoints interrogent sur les définitions de l'inceste ${ }^{47}$ et s'inscrivent dans des systèmes complexes au sein desquels les règles d'alliance répondent à de multiples considérations extérieures au champ de la parenté48. L'importance des prestations matrimoniales pour réguler ces systèmes matrimoniaux a fait l'objet d'une publication collective de Peristiany, en collaboration avec MarieElizabeth Handman ${ }^{49}$. S'opposent en ce domaine le régime de la dot, répandu dans le monde chrétien, et celui du douaire musulman, qui peut s'accompagner de prestations de la famille du mari à celle de l'épouse. Le système matrimonial, qui s'est diffusé avec le christianisme sur le modèle romain, système sacramentaire et définissant à l'inverse des prohibitions dans un large champ de consanguinité, obéit formellement à d'autres principes mais manifeste un même souci de définition de la proximité, qui, à certaines périodes, quand se relâche l'autorité ecclésiastique, voit se développer la pratique de mariages dans un degré rapproché, par ailleurs toujours possibles sous réserve de dispense religieuse.

Famille: les familles étendues sur la base d'une extension des rapports de parenté ont très tôt attiré l'attention dans les études méditerranéennes ${ }^{50}$, en particulier dans les sociétés rurales où s'observent des formes familiales communautaires, concernant

${ }^{45}$ Le »mariage arabe« ne répond pas, a priori, aux principes d'échange et de réciprocité plus ou moins immédiate que Lévi-Strauss considère comme le fondement des systèmes matrimoniaux; c'est un mariage par ailleurs au sein du groupe de filiation unilinéaire, du lignage, alors que celui-ci se définit très généralement par sa dimension exogamique dans la littérature anthropologique.

46 Le terme est inspiré du titre d'un texte de Lévi-Strauss consacré à l'étude des sociétés où il existe des groupes de filiation unilinéaire qui nouent des alliances proches ou lointaines qui définissent la composition dynamique de groupes au sein desquels la filiation ne représente qu'un principe de dévolution des biens matériels et symboliques, un >principe de droit<.

47 Le mariage entre demi-germains était ainsi permis en Grèce ancienne, et celui entre germains dans la société égyptienne ptolémaïque; c'est aussi un thème testamentaire récurrent, sinon une pratique des anciens Hébreux. En islam, l'ensemble des cousines germaines sont des conjointes potentielles pour un homme et sont épousées de manière statistiquement significative.

48 Pierre BONTE (dir.), Épouser au plus proche. Inceste, prohibitions et stratégies matrimoniales autour de la Méditerranée, Paris 1994 (Civilisations et sociétés, 89).

49 John G. PERISTIANY en collaboration avec Marie-Elizabeth HANDMAn (dir.), Le prix de l'alliance en Méditerranée, Paris 1989.

50 PeRISTIANY, Mediterranean Family Structures (voir n. 19). 
plusieurs générations, et dans le domaine foncier et politique ${ }^{51}$. C'est dans un contexte européen, distinguant nord et sud méditerranéens, que Jack Goody situe l'évolution historique des structures familiales ${ }^{52}$. Pierre Guichard, pour sa part, oppose des structures »occidentales « et »orientales « ${ }^{53}$. Dans une autre perspective, la gestion sociale et symbolique de la distinction de genre, caractérisée le plus souvent par un fort biais patriarcal et agnatique, dans le groupe domestique et dans l'ensemble de la société, a retenu l'attention de nombre d'auteurs parmi lesquels, outre Pitt-Rivers en Andalousie $^{54}$, Herzfeld en Crète, David D. Gilmore en Andalousie, Pierre Bourdieu en Kabylie, etc., ouvrant la porte à des réflexions plus générales sur l'importance de ce concept de "genre« dans les sociétés méditerranéennes.

Genre: l'organisation des valeurs du masculin et du féminin représente une des constantes les plus remarquables des sociétés méditerranéennes, qu'il s'agisse d'identifier leurs fondements structurels ${ }^{55}$ ou d'en définir l'éthos culturel ${ }^{56}$, malgré (ou en conséquence de) la forte hiérarchisation qui assure la cohérence de leurs représentations du monde ${ }^{57}$. Comme dans toutes les sociétés humaines, ces représentations du genre se développent comme une théorie plus ou moins explicite de la distinction des sexes et de la transmission d'identités sociales et symboliques à travers la reproduction sexuée de l'espèce. Les travaux sur ce thème encore peu développés dessinent quelques pistes prometteuses. Les sociétés méditerranéennes se caractérisent ainsi par l'importance accordée aux transferts de substances (sang, lait, sperme...) qu'elles partagent plus généralement avec les sociétés européennes et qui se traduit immédiatement à travers notre notion de »consanguinité«, peu pertinente pour étudier les sociétés amérindiennes par exemple. Elle pose problème aussi dans le monde musulman, où le sang est moins vecteur de transfert que d'identités individuelles. En revanche la parenté de lait met en évidence, dans des sociétés où le droit agnatique est affirmé, une transmission féminine parallèle. Les sociétés méditerranéennes oscillent généralement entre une vision monogénétique de la transmission des substances sexuées et de la conception illustrée par les thèses aristotéliciennes ${ }^{58}$, ou encore par les métaphores du labour ou de la poterie fondant le primat des substances masculines ${ }^{59}$ - et une vision duogénétique

51 Paul Henri StaHL, Household, Village and Village Confederation in the South-Eastern Europe, New York 1986 (East European monographs, 200).

52 Jack Goody, L'évolution de la famille et du mariage en Europe, Paris 1985.

53 Pierre Guichard, Structures sociales »orientales« et »occidentales« dans l'Espagne musulmane, Paris, La Haye 1977 (Civilisations et sociétés, 60).

54 Pitt-Rivers, The Fate of Shechem (voir n. 20).

55 BONTE (dir.), Épouser au plus proche (voir n. 48).

56 PITT-RIVERS, The Fate of Shechem (voir n. 20).

57 Pierre Bourdieu, Esquisse d'une théorie de la pratique, précédé de trois études d'ethnologie kabyle, Genève 1970.

${ }^{58}$ Qui repose sur le primat du sperme, dans une longue tradition méditerranéenne qui remonte à l'Égypte ancienne, associant le sperme et la moelle, la colonne vertébrale et le pénis. Pour Aristote la coction du sang produit le sperme masculin, les femmes ne pouvant réaliser à partir du sang que des coctions imparfaites, à l'origine du lait par exemple, et ne jouant de ce fait qu'un rôle second dans la conception.

59 Carol Delaney, The Seed and the Soil, Berkeley 1991 (Comparative Studies on Muslim societies, 11). 
reconnaissant les apports de chaque sexe dans la tradition hippocratique. Cette oscillation caractérise la pensée grecque et se retrouvera dans les théories arabes médiévales. Dans le monde chrétien, la vision sacramentaire d'»une même chair« (una caro fieri), déterminée par les relations sexuelles, transcende cette opposition et ouvre à de nouveaux développements.

Honneur et honte: les recherches sur la notion d'honneur caractérisent peut-être le plus spécifiquement les études méditerranéennes, et l'on peut considérer que ce concept est introduit et développé en anthropologie essentiellement à partir de ces études; elles inspirent dès le départ des recherches de terrain ${ }^{60}$ et de premières synthèses ${ }^{61}$. Les débats sur l'honneur et la honte, dans la revue »Man« durant les années 1980 et dans des travaux ultérieurs ${ }^{62}$, s'emploieront à préciser le contenu du concept et sa place dans l'interprétation d'ensemble de ces sociétés méditerranéennes. Cette place se dessine à travers une série d'oppositions qui organisent le champ de ces études: honneur et honte, honneur et grâce, honneur et baraka, intercession religieuse, etc., qui finalement se conjuguent assez étroitement avec l'opposition hiérarchique du masculin et du féminin. L'honneur est associé ainsi au sacré, dont relève ultimement le féminin; il l'est aussi au statut, selon une formule souvent citée: l'honneur se gagne par les hommes et se perd par les femmes; il l'est enfin aux identités constitutives des individus et des groupes en tant que capital symbolique qui se manifeste à l'occasion de l'alliance de mariage dont il légitime les règles, de la manipulation de la violence, du patronage politique, etc.

Traitement de la violence et système vindicatoire: cette thématique de la recherche, étroitement associée à la précédente, s'est d'abord intéressée aux cycles de violence prenant la forme de feuds ou de vendettas qui sont particulièrement remarquables dans certaines sociétés méditerranéennes. Deux auteurs proposent des synthèses sur la question: Jack Black-Michaud et Christopher Boehm ${ }^{63}$. Plusieurs pistes ont été suivies. La plus classique est l'exploitation des codes locaux ( $q \bar{a} n \bar{n} n$ ), écrits ou oraux, qui codifient et enregistrent la dynamique du système. D'autres auteurs se sont penchés sur les effets de l'institution comme instrument de gestion du politique, ou encore comme manipulation mafieuse du lien social. D'autres enfin se sont intéressés à la forte ritualisation qui accompagne la gestion de la violence, les rituels du meurtrier par exemple. Plus récemment, d'autres formes de violence ont attiré l'attention des chercheurs, suscitant un nouvel intérêt dans les contextes de contacts culturels - entre autres ma-

60 Pitt-Rivers, The People of the Sierra (voir n. 20); John K. CAmpBell, Honour, Family and Patronage. A Study of Institutions and Moral. Values in a Greek Mountain Community, Oxford 1964.

61 Peristiany (dir.), Honour and Shame (voir n. 18).

62 Raymond JAMOUS, Honneur et Baraka. Les structures sociales traditionnelles dans le Rif, Paris 1981; David D. GILMORE, Honor and Shame and the Unity of the Mediterranean, Washington DC 1987; PERISTIANY, PITT-RIVERS (dir.), Honor and Grace (voir n. 18).

63 Jack Black-Michaud, Cohesive Force. Feud in the Mediterranean and the Middle East, Oxford 1975; Christopher BoEHM, Blood revenge. The Anthropology of Feuding in Montenegro and Other Tribal Societies, Philadelphie 1984. 
riage forcé, sororicide, crimes d'honneur, etc. - ou dans les situations de guerre civile, dans les Balkans ou au Proche-Orient.

Patronage et clientélisme: l'étude des mécanismes de protection s'inscrit aussi en continuité avec la thématique de l'honneur. C'est plus particulièrement le cas de leur institutionnalisation en tant que relation patron/client dont Ernest Gellner et John Waterbury offrent une première approche comparative dans le monde musulman ${ }^{64}$. Les travaux de terrain se sont multipliés sur les contextes économiques et sociaux de ces relations de patronage, à propos des phénomènes mafieux par exemple ${ }^{65}$, ainsi que sur leur dimension politique. Ces relations sont susceptibles de favoriser des formes d'organisation politique plus ou moins stables sur une base tribale par exemple, généralisant et institutionnalisant la protection ${ }^{66}$, ou de s'inscrire dans l'appareil étatique en définissant le fonctionnement des institutions locales »caciquat" et en alimentant le clientélisme électoral.

Formes de religiosité: plusieurs problématiques sur ce thème ont été développées dans le contexte méditerranéen. Les premiers travaux ont exploré les thèmes de la magie et de la sorcellerie, largement exploités déjà dans d'autres parties du monde ${ }^{67}$. Les recherches plus récentes s'emploient plutôt à mettre l'accent sur les fondements symboliques des faits religieux ${ }^{68}$. Le rapprochement entre ce qui est généralement intitulé le »culte des saints«, saints médiateurs des relations avec le surnaturel et détenteurs d'un pouvoir d'intercession efficace, dans le monde musulman et chrétien, à l'interface des formes de religiosité populaire et savante, a suscité des travaux intéressants ${ }^{69}$, qui n'ont pas exploré toutes les dimensions comparatives de cette riche problématique, alors que se multipliaient parallèlement les recherches de terrain ${ }^{70}$ ouvrant sur des essais de synthèse ${ }^{71}$. La dimension festive de la religiosité a aussi attiré très tôt l'attention ${ }^{72}$ et se prolonge de nos jours avec la multiplication des travaux sur les pèle-

64 Ernest Gellner, John Waterbury (dir.), Patrons and Clients in Mediterranean Societies, Londres 1977.

65 Anton BLOK, The Mafia in a Sicilian Village 1860-1960. A Study of Violent Peasant Entrepreneurs, Oxford 1974; voir aussi Jane SCHNEIDER, Peter SCHNEIDER, Revisible Destiny. Mafia, Antimafia and the Struggle for Palermo, Berkeley 2003.

66 Pierre BONTE, L'émirat de l'Adrar. Harîm, compétition et protection dans une société tribale saharienne, Paris 2008.

67 Juan Caro BAROJA, Les sorcières et leur monde, Paris 1972; Ernesto di MARTINO, Italie du Sud et magie, Paris 1963.

${ }^{68}$ Dale F. Eickelman, Moroccan Islam. Tradition and Society in a Pilgrimage Center, Austin 1976 (Modern middle east series, 1).

${ }^{69}$ Peter Brown jouant un rôle de précurseur voir ID., Le culte des saints: son essor et ses fonctions dans le monde chrétien, Paris 1984; ID., La société et le sacré dans l'Antiquité tardive, Paris 1985.

70 Jeremy BoISSEVAIN, Saints and Fireworks. Religion and Politics in Rural Malta, Londres 1969 (Monographs on social anthropology, 30).

71 Mohamed Kerrou, L'autorité des saints. Perspective historique et socio-anthropologique en Méditerranée, Paris 1998.

72 Louis DumOnT, La tarasque: essai de description d'un fait social d'un point de vue ethnographique, Paris 1951 (L'espèce humaine, 8); Julio CARO BAROJA, Le carnaval. Traduit de l'espagnol par Sylvie SESÉ-LÉGER, Paris 1979 ( $1^{\text {re }}$ éd. 1965). 
rinages ${ }^{73}$ et autres phénomènes festifs ${ }^{74}$. Ceux-ci ont aussi été étudiés hors contexte religieux (carnaval, charivari, etc.) en intégrant des manifestations contemporaines telles les rencontres de football.

Religions révélées, mythes d'origine et rituels: s'alimentant d'une relecture des textes avec les méthodes de l'anthropologie ${ }^{75}$, la réflexion sur les mythes fondateurs des religions révélées a contribué à de nouvelles études des grands rituels qu'elles ont développés, le rituel sacrificiel par exemple 76 qui s'organise en islam autour de la référence au récit du sacrifice du fils demandé par Dieu à Abraham. Ce rituel représente une approche privilégiée dans des travaux comparatifs sur les trois monothéismes qui l'inscrivent dans leur démarche théologique sous des formes diversifiées. Ces recherches testamentaires ont aussi permis d'aborder l'étude des systèmes de classification qui organisent les représentations du monde ${ }^{77}$, ouvrant sur des perspectives comparatives qui débordent l'aire concernée mais éclairent sur les fondements cognitifs et symboliques des sociétés méditerranéennes.

Ces différents traits culturels qui dessinent, d'une manière que nous avons appelée »agrégative«, un champ d'études méditerranéennes n'acquièrent pas immédiatement, dans la perspective de la définition d'un objet d'étude spécifié, une valeur heuristique du fait de ce seul rapprochement.

\section{BILAN D’UNE ANTHROPOLOGIE DE LA MÉDITERRANÉE}

Constatons d'abord que l'institutionnalisation de ce champ d'études méditerranéennes est restée limitée. Les travaux se sont développés initialement à l'occasion de conférences internationales dont sont issus la plupart des premiers ouvrages de synthèse cités et que l'on peut considérer dans cette mesure comme fondateurs. La première eut lieu en 1959 à l'initiative de Pitt-Rivers à Burg Wartenstein, en Autriche, la seconde en 1961 à Athènes autour de Peristiany; viendra ensuite le colloque de Canterbury en 1966. Ce n'est que plus tardivement que naîtront des journaux spécialisés. On peut citer »Peuples méditerranéens/Mediterranean Peoples«, revue interdisciplinaire fondée en 1974, et, de contenu plus anthropologique, »Mediterranean/Méditerranéennes« fondée en 1990 par Kenneth Brown, et le »Journal of Mediterranean Studies« en 1991

73 Jill Dubisch, In a Different Place. Pilgrimage, Gender and Politics in a Greek Island shrine, Princeton 1995; Elizabeth CLAVERIE, Les guerres de la Vierge. Une anthropologie des apparitions, Paris 2003.

74 En ce qui concerne la fête musulmane de l' îd al-kabir voir Abdellah HAMMOUDI, La victime et ses masques, Paris 1988; Anne-Marie BrIESEBARRE, La fête du mouton. Un sacrifice musulman dans l'espace urbain, Paris 1998.

75 Edmund LEACH, L'unité de l'homme et autres essais, Paris 1980, regroupant sous un titre énigmatique des extraits d'un ouvrage publié en 1969 sous le titre »Genesis as Myth « et d'une autre publication avec Alan AYCOK, Structuralist Interpretations of Biblical Myth, Cambridge 1983.

76 Pierre Bonte, Anne-Marie Brisebarre, Édouard Conte, Sacrifices en islam. Espaces et temps d'un rituel, Paris 1999.

77 Mary Douglas, De la souillure, Paris 1971. 
par Paul San Cassia ${ }^{78}$. Les associations scientifiques, telle ADAM, créée en France en 2001, sont plus rares encore.

Ce faible développement institutionnel des études méditerranéennes traduit un certain désintérêt pour l'élaboration d'un cadre théorique partagé, et doit être rapproché de la production limitée de travaux de synthèse anthropologiques. Le questionnement sur la Méditerranée des anthropologues, s'il relève d'un pragmatisme raisonné et revendique un certain empirisme, n'en mérite pas moins l'attention.

Un problème complexe est ainsi celui que soulève la définition des »frontières« de l'espace concerné par les études méditerranéennes, et parallèlement la mise en évidence de distinctions régionales opérant en leur sein. Une histoire plurimillénaire a contribué à la diffusion de traits >civilisationnels`, entendus ici en un sens proche de »culturels«, mais fortement historicisés, largement diffusés dans l'aire géographique méditerranéenne et caractéristiques, sur le plan des comportements, des institutions et des valeurs, des sociétés qui y sont inscrites. Les fondements gréco-romains ou encore judéo-chrétiens des sociétés modernes continuent à être invoqués jusqu'à nos jours et suscitent même des débats passionnés comme le manifestent les discours sur les fondements chrétiens de l'Europe ou ceux sur l'intégration européenne de la Turquie que nous avons déjà évoqués.

Ces mouvements de brassage, accompagnés de déplacements de population, de réorganisation des échanges et de diffusion de traits institutionnels et culturels, se sont poursuivis jusqu'à l'époque contemporaine à travers les phénomènes hégémoniques de diffusion religieuse ${ }^{79}$, en particulier de christianisation et d'islamisation ${ }^{80}$, et du fait de la colonisation économique et politique qui se généralise sur la rive méridionale au tournant du XIX ${ }^{\mathrm{e}}$ et du XX⿳⺈冂大 siècle, seule la Turquie préservant son indépendance malgré le dépècement de l'Empire ottoman au lendemain de la Première Guerre mondiale. La

78 On retrouve en France la référence à la Méditerranée dans l'intitulé de la »Revue des études musulmanes et méditerranéennes«, ancienne »Revue de l'Occident musulman et méditerranéen «.

79 La christianisation des sociétés méditerranéennes est achevée après la conversion massive de l'Empire romain et se prolongera jusqu'à la diffusion de l'islam au sein de l'Empire byzantin et des États de la Méditerranée occidentale. Elle est caractérisée par le pouvoir législatif et politique des Églises qui, au delà de leurs différenciations historiques anciennes, contribuent à une forte normalisation des comportements juridiques et politiques et à la reconnaissance d'institutions canoniques dans le domaine du mariage, du baptême, du parrainage, etc. L'islamisation sera tout aussi rapide jusqu'à ce que s'établissent entre le monde chrétien et le monde de l'islam des frontières qui connaîtront des réajustements périodiques, à l'occasion des croisades par exemple, ou encore dans la péninsule Ibérique et dans les Balkans, ceci jusqu'à la période contemporaine avec le déclin des christianismes orientaux ou le développement de la diaspora musulmane en Europe par exemple. Le droit musulman (fiqh), avec ses variations d'écoles, s'imposera au sein de la umma, communauté musulmane, ainsi que des pratiques sociales (mariage, parenté de lait), religieuses (soufisme) et politiques, celles-ci s'organisant longtemps dans le monde sunnite, le plus largement représenté, autour de la question du califat, de la direction centralisée de la communauté, question évacuée avec la formation de l'Empire ottoman.

80 Sans oublier la récente réinscription du judaïsme dans l'aire méditerranéenne sous une forme nouvelle, celle de la revendication sioniste de la création d'un État-nation. 
colonisation et les nouvelles formes de globalisation qui la suivent brouillent les frontières humaines, culturelles et politiques, mais elles créent aussi de nouvelles frontières moins géographiques qu'idéologiques, que l'on résume parfois sous l'intitulé du »choc des civilisations«. Il en résulte de nouvelles formes de conflictualité, qui ont pris un tour dramatique depuis le 11 septembre 2001, mais qui présentent aussi de multiples facettes: compétition pour le contrôle des ressources et la redistribution des richesses, affrontements politiques et juridiques à propos du statut personnel et des droits de la femme, différends sur les modèles laïcs et démocratiques de la gouvernance, etc.

La colonisation, puis la globalisation, n'ont pas eu seulement pour effet d'inscrire les sociétés colonisées dans le moule contraignant des sociétés industrielles et capitalistes occidentales, elles entraînent aussi une réélaboration des modèles culturels qui s'appuient sur des traits hérités mais réinterprétés dans le contexte contemporain avec de nouvelles significations. Le cas de la >cuisine méditerranéenneく est à cet égard exemplaire: il ne s'agit pas seulement d'une nouvelle forme d'uniformisation culturelle - associée pour une part au tourisme de masse - à laquelle correspondent des valeurs esthétiques, gastronomiques, certes, elle présente aussi les caractères d'un art de vivre, et même d'un bien-vivre quand cette >cuisine méditerranéenne` se voit attribuer des qualités diététiques exceptionnelles ${ }^{81}$.

Ces brassages et conflits humains et culturels sont l'arrière-plan de la question des frontières, externes et internes, de la Méditerranée des anthropologues. Le problème se pose de manière différente selon qu'il s'agit des frontières >orientales` (africaines et asiatiques) ou >européennes〈. L'usage de l'arabe et la présence d'un ordre tribal présentant des traits relativement convergents ${ }^{82}$ nous conduiraient à situer la limite africaine au sud du Sahara. Mais faudrait-il alors considérer comme >méditerranéenneく la péninsule arabique en faisant abstraction de ses contacts anciens et étroits avec les côtes africaines et asiatiques, de l'Afrique de l'Est à l'Inde et à l'Asie du Sud-Est? Où arrêter ces frontières méditerranéennes au Moyen-Orient: à l'Irak, à l'Iran, voire au Pakistan, là où se conjuguent islam et affiliation tribale? Mais on ne peut comprendre les évolutions qu'ont connues ces régions du Moyen-Orient sans tenir compte des apports de l'Asie centrale, sur le plan humain, à travers les migrations des populations turco-mongoles, comme sur le plan culturel. Dominant durant des siècles le bassin méditerranéen, les Ottomans ont été par ailleurs les principaux vecteurs de la diffusion de ces traditions turco-mongoles depuis l'Asie centrale.

81 Pour être exhaustif en ce domaine, rappelons le rôle des colonisations portugaise et espagnole qui ont contribué au transfert de certains traits culturels méditerranéens en Amérique latine où on les retrouve dans les cultures latino-américaines. Il s'agit d'apports techniques et économiques (mesta, système de transhumance), sociaux et politiques (patronage et caciquat), religieux (confréries et culte des saints), etc.

82 Mais aussi des distinctions remarquables. Tout en partageant le mode de vie des sociétés bédouines de l'ouest-saharien, les Touaregs du Sahara central organisent leurs tribus en se référant à une filiation utérine, à des ancêtres féminins. Il est possible que cela ait été aussi le cas historiquement des populations berbères du nord de l'Afrique, ainsi que des anciens Nubiens qui occupaient le nord du Soudan contemporain et le sud de l'Égypte - on retrouve ce même trait chez les Béja contemporains répartis entre l’Égypte et le Soudan. 
Mais alors, pour en revenir aux limites >européennes«, où situer la frontière dans les Balkans? Où cette présence ottomane a-t-elle des conséquences de nos jours? Faut-il en Europe continentale tracer la frontière entre langues romane et germanique, finalement deux rameaux des langues indo-européennes? Il est clair que ces interrogations ne sont pas susceptibles de déboucher sur des réponses définitives.

Celles que l'on peut apporter sont à rapprocher des réponses que l'on peut donner aux interrogations soulevées par l'identification de frontières internes. L'essentiel est, nous semble-t-il, d'éviter l'écueil des limites rigides et définitives, qu'elles soient civilisationnelles (Occident/Orient), religieuses (christianisme/islam) ou encore culturelles et linguistiques (Indo-européens/Sémitiques) dont l'historien, et l'orientaliste, peuvent se satisfaire, pour peu qu'ils fassent preuve d'un certain relativisme et tiennent compte du mouvement du temps, des migrations des hommes, des biens et des idées, du jeu des influences, de l'établissement des hégémonies et de leur déclin. C'est la Méditerranée de Philippe II -, objet de l'ouvrage exemplaire de Braudel qui porte un regard globalisant sur le champ d'études - qui peut, qui doit, s'accommoder des ruptures économiques, politiques, culturelles, organisant alors cet objet ${ }^{83}$. Dans sa complémentarité avec la démarche historique, et sans être condamnée à la synchronie, l'approche anthropologique va être amenée à définir ces ruptures, ces frontières régionales, en prenant en compte la définition des paradigmes de ses recherches, en fonction de la nature des questions soulevées et des objets dont elle traite.

Dans la perspective comparative, cette approche anthropologique doit ainsi prendre en considération un autre type de problème encore: celui de l'échelle des objets dont elle traite, étant entendu que ses terrains ont généralement une dimension monographique. Les recherches ont été menées dans le cadre d'un village, d'une tribu ou d'une fraction de tribu; il s'agit parfois d'une microrégion ou d'un groupe ethnique lui-même inscrit à une échelle régionale restreinte ${ }^{84}$. Cette démarche est justifiée théoriquement par les anthropologues en référence à la notion maussienne de »fait social total«, qui légitime une approche de la totalité d'une société à travers une institution particulière, ou encore à celle de "patterns of culture « introduite par Alfred Kroeber, qui déduit la cohérence d'un modèle culturel à partir de ses configurations particulières ${ }^{85}$. De ce fait, le travail comparatif vise moins à définir des ensembles différenciés plus ou moins vastes qu'à mettre en évidence les effets des variations de traits particuliers, remarquables localement, sur l'organisation de ces stotalités sociales ou culturelles. C'est ainsi qu'au Liban, par exemple, la forte diversité des appartenances religieuses communautaires, institutionnalisées au sein de l'État libanais, conduira à la multiplication des études sur chrétiens et musulmans. Une autre opposition souvent explorée est celle

83 Fernand BRAUDEL, La Méditerranée et le monde méditerranéen à l'époque de Philippe II, Paris 1949.

${ }^{84}$ C'est le cas des recherches menées par exemple sur des populations nomades ou transhumantes, pastorales, insérées dans les milieux régionaux des Balkans (Saracatsans de Grèce et Bulgarie) ou en Turquie (Yörük).

85 Cette approche de la culture chez Kroeber est un des fondements de la version américaine de la théorie des »aires culturelles«. 
entre nomades et sédentaires, qui a été parfois envisagée à des échelles plus larges ${ }^{86}$. En définitive, cependant, les travaux anthropologiques, à quelques exceptions près comme la méthode cartographique par exemple 87 -, ne s'intéresseront pas plus aux convergences et divergences définissant d'éventuels ensembles régionaux qu'ils ne se sont risqués à établir de grandes synthèses concernant les traits caractéristiques de l'aire méditerranéenne dans sa totalité.

\section{PERSPECTIVES}

Le bilan des recherches sur lesquelles pourrait se fonder une anthropologie de la Méditerranée reflète plus cette agrégation empirique de travaux et de traits sociaux et culturels que nous venons de résumer qu'il ne définit un champ institutionnel et scientifique cohérent et partagé. On ne doit pas s'en étonner si l'on considère l'imprécision de l'objet et la remise en question, générale de nos jours, de la problématique des aires culturelles, parallèlement à la critique d'autres notions classificatoires comme celles d'»ethnie« ou de »tribu«. Une réflexion sur la Méditerranée des anthropologues, cependant, n'a pas seulement un intérêt du point de vue des développements épistémologiques de la discipline, ne serait-ce que du fait de la richesse des significations accordées à cette notion »Méditerranée« dans le monde contemporain, qu'il s'agisse de l'associer ou de la situer par rapport à la construction européenne ou de définir des options politiques et stratégiques, qu'on les conçoive en terme d'expansion des valeurs occidentales, ou sous celui de »choc des civilisations«.

Deux approches apparaissent néanmoins possible du point de vue de l'anthropologue, qui se définissent entre deux pôles dont l'un correspond à la recherche de permanences historiques et structurales, et l'autre à la production de nouvelles formes et de nouveaux objets en tant que conséquence de processus que nous appellerons métaphoriquement de >métissage

Les thématiques en fonction desquelles se sont organisées ces études méditerranéennes sont plus ou moins spécifiques à celles-ci, jamais exclusives, et elles présentent, dans leur contenu, des variations là encore plus ou moins marquées: les problématiques de l'honneur par exemple ne se présentent pas dans les mêmes termes et ne se traduisent pas par les mêmes représentations et pratiques en Italie et en Grèce, au Maghreb et au Machreq - même si l'on identifie des constantes et des principes identiques.

86 C'est le cas de l'ouvrage de Jacob Black-Michaud, Cohesive Force: Feud in the Mediterranean and the Middle East, Oxford 1975, traitant des phénomènes de feuds et de vendettas à l'échelle méditerranéenne. La thèse est que ces phénomènes sont, contrairement aux idées reçues, plus violents et pérennes chez les sédentaires (Rif, Albanie) que chez les bédouins nomades.

87 Illustrée par Christian Bromberger et Jean-Pierre Digard qui l'appliquent à l'Iran. Associée aux travaux sur les aires culturelles et aux thèses diffusionnistes, elle se révèle surtout efficace en ce qui concerne la répartition des traits matériels (techniques, habitat). Voir Christian BROMBERGER, Cartes ethnographiques, dans: Pierre BONTE, Michel IZARD (dir.), Dictionnaire de l'ethnologie et de l'anthropologie, Paris 1991, p. 127-129. 
J'ai déjà noté des réenchainements et des recoupements en énumérant des thèmes que j'ai considérés par ailleurs comme rassemblés sous une forme agrégative dans les études méditerranéennes. On peut ainsi trouver des continuités entre les analyses concernant les points suivants: alliance de mariage (inceste, stratégies matrimoniales); honneur (honte, statut, prestige); protection (patronage); système vindicatoire (manipulation de la violence); structure familiale (extension de la parenté); filiation (solidarités intergénérationnelles et lien social). La notion de genre, la construction et le traitement du masculin et du féminin fournissent un fil partiel qui permet de dérouler la trame de ces valeurs et de ces institutions. D'autres réenchaînements sont de même identifiables, dans le domaine du religieux par exemple.

Sur cette base, l'anthropologue est susceptible de produire des hypothèses quant à l'existence de permanences qui rendent possible le rapprochement de sociétés séparées dans le temps et dans l'espace. Que l'on parle alors, après Claude Lévi-Strauss, de principes structuraux ou, suivant Louis Dumont, de systèmes de valeur, relève de l'appareil théorique et méthodologique de référence, et ne remet pas en cause la démarche elle-même qui vise à l'approfondissement de la comparaison: l'objectif pour l'anthropologue est autant d'identifier des divergences que de constater des convergences.

À titre d'exemple de la démarche, j'ai personnellement développé quelques hypothèses de cet ordre que je souhaiterais présenter ici. On peut ainsi rapprocher les systèmes de parenté et d'alliance de la Grèce ancienne, le système athénien du moins, et ceux du monde arabe, et noter que le système spartiate que dénonçait Aristote pour les risques de gynécocratie et de tyrannie dont il est porteur selon lui, se rapprocherait alors de celui que l'on observe chez les Touaregs. Ces observations iraient dans le sens de l'identification d'une aire méditerranéenne de la parenté, présentant des structures particulières ${ }^{88}$, ce qui n'implique pas que tous les systèmes de parenté et d'alliance observés dans ces régions relèvent de ces structures. Les données arabes et touaregs, quant à elles, peuvent être traitées; nous avons tenté de montrer la pertinence de cette hypothèse de travail ${ }^{89}$, comme des variations sur le même principe de distinction des sexes et d'organisation de la reproduction sexuée à travers les régimes de filiation et d'alliance. Les textes testamentaires, plus particulièrement le sacrifice demandé par Dieu à Abraham de son fils, en offrent des représentations fondatrices, traitées différemment selon les religions révélées en fonction de leur conception et de leur pratique

88 La notion d'»aire« n'est pas entendue ici dans le sens d'»aire culturelle« mais plutôt dans la perspective introduite par Lévi-Strauss dans son étude des structures élémentaires de la parenté. Il montre que les systèmes d'échange généralisé s'organisent autour d'un axe traversant l'Asie occidentale alors que les systèmes d'échange restreint le sont sur un axe traversant l'Asie du Sud-Est et l'Austronésie. Les connotations géographiques et culturelles ne sont pas ici déterminantes, il s'agit de convergences structurales que ne commentera pas l'auteur par ailleurs.

89 Pierre BONTE, Les lois du genre. Approches comparatives des systèmes de parenté arabes et touaregs, dans: Jean-Luc JAMARD, Emmanuel TERRAY, Margarita XANTHAKOU (dir.), En substances. Textes pour Françoise Héritier, Paris 2000, p. 135-156. 
de la filiation ${ }^{90}$. Les représentations du processus de reproduction biologique, empruntées par les musulmans aux savants grecs, développent par ailleurs une même variation significative entre les théories duogénétiques hippocratiques et les théories monogénétiques que développe Aristote. L'hypothèse d'un traitement spécifié de la notion de genre comme principe structurant l'ordre social et/ou comme valeur fondatrice de sociétés et cultures particulières, a une certaine efficacité pour développer des problématiques recoupant ces thèmes méditerranéens; et elle pourrait être testée en d'autres domaines: choix du conjoint, statut du féminin, association au sacré, etc. ${ }^{91}$.

La Méditerranée des anthropologues apparaît de ce point de vue comme une construction ouverte, issue d'une approche déductive structurale, produisant des hypothèses théoriques qui peuvent être renouvelées, voire abandonnées si leur pertinence s'épuise ou apparaît douteuse.

Une autre modalité de construction de l'objet »Méditerranée« l'envisage différemment, de manière inductive et empirique, envisageant la continuité et l'homogénéisation des traits sociaux et culturels, à travers l'émergence de nouvelles formes sociales culturelles résultant des contacts établis entre les cultures et les sociétés. La métaphore du »métissage« pour désigner ces processus a été utilisée par d'autres auteurs, Herzfeld par exemple, qui a lui-même emprunté le terme à Lee Drummond tentant de rendre compte de ce qu'il appelle le cultural continuum. Certes, comme l'observent Christian Bromberger et Jean-Yves Durand ${ }^{92}$, les sociétés méditerranéennes ne sont pas des »sociétés métisses«, simples produits des flux d'échanges et des contacts, mais si l'on s'en tient à l'expression simultanée de la continuité et de la rupture génétiques dont le terme »métissage« porte sens, la métaphore nous semble recevable et féconde. Pour citer un exemple récent, elle pourrait s'appliquer au brillant ouvrage consacré par Jocelyne Dakhlia aux effets de langue ${ }^{93}$, de communication uniformisante, qu'elle a étudiés dans le bassin méditerranéen. Dans le même domaine linguistique, mais sous une tout autre forme, on pourrait évoquer aussi le mouvement de dialectisation du latin à l'origine des langues romanes. Mais ces »métissages« sont observables aussi en d'autres domaines.

Ainsi le système de parenté et d'alliance de mariage que va progressivement contrôler l'Église catholique pour affirmer son rôle social et moral, porte la marque du développement du christianisme au sein de l'Empire romain en reprenant le système de prohibitions matrimoniales au septième degré de collatéralité que suivait la société romaine; l'alliance présentera la même tension que dans l'Empire romain entre l'extension des interdits et le mariage proche. De même, l'islamisation s'accompagne souvent de l'adoption du »mariage arabe«, qui n'est pourtant pas prescrit par l'islam, y compris dans des sociétés dont le système matrimonial reposait sur des principes exo-

90 Pierre Bonte, Les figures d'Abraham et la question de la filiation dans les religions monothéistes moyen-orientales, dans: BONTE, PORQUERÉS Y GÉNÉ, WILGAUX (dir.), L'argument de la filiation (voir n. 11).

91 Pierre BONTE, L'échange est-il un universel?, dans: L'Homme. Revue française d'anthropologie 154-155 (2000b) [= Question de parenté], p. 39-65.

92 Dans Dionigi, BloK, Bromberger (dir.), Anthropologie (voir n. 23).

93 Jocelyne DAKHLIA, Lingua franca. Histoire d'une langue métisse en Méditerranée, Paris 2008. 
gamiques contradictoires avec celui-ci94. La transcription du droit romain dans les codes, civils ou non, correspond aux mêmes phénomènes. Les transferts intellectuels et scientifiques du monde grec au monde arabe, et pour une part de celui-ci au monde médiéval occidental, traduisent des processus du même ordre. D'Aristote à Averroes puis à Saint-Thomas d'Aquin, ces transferts ne sont pas seulement cumulatifs. Ils impliquent des modifications et des transformations qui justifient l'emploi de la métaphore du »métissage«, rendant compte de cette transmission à la fois cumulative et créatrice. C'est là un champ quasi inépuisable qui s'ouvre aux études méditerranéennes, débordant les enjeux académiques pour déboucher sur une interrogation sur les identités contemporaines.

Finalement, les études méditerranéennes se présentent comme un vaste champ d'études comparatives, qui peuvent se développer à des échelles variables, allant du local au général, offertes aux observations des anthropologues. Ceux-ci ont pu parfois y déceler des régularités et des constantes, inséparables d'une histoire, partagée plutôt que commune, dont la dimension ne peut être ignorée d'eux, qu'il s'agisse des problématiques ou des méthodes. Ces études révèlent aussi des variations significatives justifiées là encore par la diversité des parcours historiques et par la cohérence particulière des sociétés et des cultures. S'il faut abandonner l'idée d'identifier les traits d'une aire culturelle, on pourrait plus simplement constater que ces régularités et ces variations forment plus ou moins un système. Il nous faut aussi reconnaître que ces systèmes sont abordés différemment selon les paradigmes des écoles et des auteurs, enrichissant une recherche qui tend à se distancier de ses présupposés originaux avec le déplacement du regard porté par les anthropologues indigènes sur leur propre société, mais aussi sur le fait méditerranéen.

94 Altan Gokalp a étudié cette diffusion de mariage arabe dans le monde turc, Altan GOKALP, »Mariages de parents«: entre l'échange généralisé et le mariage parallèle. Le cas de la Turquie, dans: Pierre BONTE (dir.), Épouser au plus proche (voir n. 48), p. 439-452. On observe la même évolution de systèmes d'alliances fondés sur des interdits claniques, prohibant le renouvellement générationnel de l'échange dans une partie de l'Afrique de l'Est islamisée (Tchad, Soudan, Somalies). 